\title{
A study of fractional-order coupled systems with a new concept of coupled non-separated boundary conditions
}

\author{
Hamed H Alsulami ${ }^{1}$, Sotiris K Ntouyas ${ }^{1,2}$, Ravi P Agarwal ${ }^{3}$, Bashir Ahmad ${ }^{1 *}$ and Ahmed Alsaedi ${ }^{1}$
}

\author{
"Correspondence: \\ bashirahmad_qau@yahoo.com \\ ${ }^{1}$ Nonlinear Analysis and Applied \\ Mathematics (NAAM) - Research \\ Group, Department of Mathematics, \\ Faculty of Science, King Abdulaziz \\ University, P.O. Box 80203, Jeddah, \\ 21589, Saudi Arabia \\ Full list of author information is \\ available at the end of the article
}

\begin{abstract}
In this paper, we introduce a new concept of coupled non-separated boundary conditions and solve a coupled system of fractional differential equations supplemented with these conditions. The existence results obtained in the given configuration are not only new but also yield some new special results corresponding to particular values of the parameters involved in the problem. For the illustration of the existence and uniqueness result, an example is constructed.
\end{abstract}

MSC: 34A08; 34B15

Keywords: fractional differential systems; non-separated boundary conditions; existence; fixed point

\section{Introduction}

Fractional differential equations have gained considerable importance due to their varied applications in many problems of physics, chemistry, biology, applied sciences and engineering. The tools of fractional calculus are found to be of great support in developing a more realistic mathematical modeling of the applied problems in terms of fractional differential equations. Fractional-order models are regarded as better than the classical ones (based on differential equations) as fractional derivatives can take care of the hereditary properties of materials and processes involved in the problem at hand. For details and explanations, we refer the reader to the texts [1-4]. In particular, a great interest has been shown by many authors in the subject of fractional-order boundary value problems (BVPs), and a variety of results for BVPs equipped with different kinds of boundary conditions have been obtained, for instance, see [5-13] and the references cited therein.

Coupled systems of fractional-order differential equations constitute an interesting and important field of research in view of their applications in many real world problems such as anomalous diffusion [14], disease models [15-17], ecological models [18], synchronization of chaotic systems [19-21], etc. For some theoretical works on coupled systems of fractional-order differential equations, we refer the reader to a series of papers [22-27].

In this paper, we consider a new boundary value problem of coupled Caputo type fractional differential equations:

$$
\begin{cases}{ }^{\mathrm{c}} D^{\alpha} x(t)=f(t, x(t), y(t)), & t \in[0, T], 1<\alpha \leq 2 \\ { }^{\mathrm{c}} D^{\beta} y(t)=g(t, x(t), y(t)), & t \in[0, T], 1<\beta \leq 2,\end{cases}
$$

(c) The Author(s) 2017. This article is distributed under the terms of the Creative Commons Attribution 4.0 International License (http://creativecommons.org/licenses/by/4.0/), which permits unrestricted use, distribution, and reproduction in any medium, provided you give appropriate credit to the original author(s) and the source, provide a link to the Creative Commons license, and indicate if changes were made. 
subject to the following non-separated coupled boundary conditions:

$$
\begin{cases}x(0)=\lambda_{1} y(T), & x^{\prime}(0)=\lambda_{2} y^{\prime}(T), \\ y(0)=\mu_{1} x(T), & y^{\prime}(0)=\mu_{2} x^{\prime}(T),\end{cases}
$$

where ${ }^{\mathrm{c}} D^{\alpha},{ }^{\mathrm{c}} D^{\beta}$ denote the Caputo fractional derivatives of order $\alpha$ and $\beta$, respectively, $f, g:[0, T] \times \mathbb{R} \times \mathbb{R} \rightarrow \mathbb{R}$ are appropriately chosen functions, and $\lambda_{i}, \mu_{i}, i=1,2$, are real constants with $\lambda_{i} \mu_{i} \neq 1, i=1,2$.

Here we emphasize that our problem is new in the sense of non-separated coupled boundary conditions introduced here. To the best of our knowledge, fractional-order coupled system (1.1) has yet to be studied with the boundary conditions (1.2). In consequence, our findings of the present work will be a useful contribution to the existing literature on the topic. The existence and uniqueness results for the given problem are new, though they are proved by applying the well-known method based on Banach's contraction principle and Leray-Schauder's alternative.

The rest of the contents of the paper is organized as follows. In Section 2, we recall some basic definitions of fractional calculus and present an auxiliary lemma, which plays a pivotal role in obtaining the main results presented in Section 3. We also discuss an example for illustration of the existence-uniqueness result. The paper concludes with some interesting observations.

\section{Preliminaries}

First of all, we recall some basic definitions of fractional calculus.

Definition 2.1 The fractional integral of order $r$ with the lower limit zero for a function $f$ is defined as

$$
I^{r} f(t)=\frac{1}{\Gamma(r)} \int_{0}^{t} \frac{f(s)}{(t-s)^{1-r}} d s, \quad t>0, r>0,
$$

provided the right-hand side is point-wise defined on $[0, \infty)$, where $\Gamma(\cdot)$ is the gamma function, which is defined by $\Gamma(r)=\int_{0}^{\infty} t^{r-1} e^{-t} d t$.

Definition 2.2 The Riemann-Liouville fractional derivative of order $r>0, n-1<r<n$, $n \in \mathbb{N}$, is defined as

$$
D_{0+}^{r} f(t)=\frac{1}{\Gamma(n-r)}\left(\frac{d}{d t}\right)^{n} \int_{0}^{t}(t-s)^{n-r-1} f(s) d s
$$

where the function $f(t)$ has an absolutely continuous derivative up to order $(n-1)$.

Definition 2.3 The Caputo derivative of order $r$ for a function $f:[0, \infty) \rightarrow \mathbb{R}$ can be written as

$$
{ }^{\mathrm{c}} D^{r} f(t)=D_{0+}^{r}\left(f(t)-\sum_{k=0}^{n-1} \frac{t^{k}}{k !} f^{(k)}(0)\right), \quad t>0, n-1<r<n .
$$


Remark 2.4 If $f(t) \in C^{n}[0, \infty)$, then

$$
{ }^{\mathrm{c}} D^{r} f(t)=\frac{1}{\Gamma(n-r)} \int_{0}^{t} \frac{f^{(n)}(s)}{(t-s)^{r+1-n}} d s=I^{n-r} f^{(n)}(t), \quad t>0, n-1<r<n .
$$

Now we present an auxiliary lemma which plays a key role in the sequel.

Lemma 2.5 Let $\phi, h \in C([0, T], \mathbb{R})$ and $\lambda_{i} \mu_{i} \neq 1, i=1,2$. Then the solution of the linear fractional differential system

$$
\left\{\begin{array}{lc}
{ }^{\mathrm{c}} D^{\alpha} x(t)=\phi(t), & t \in[0, T], 1<\alpha \leq 2, \\
{ }^{\mathrm{c}} D^{\beta} y(t)=h(t), & t \in[0, T], 1<\beta \leq 2, \\
x(0)=\lambda_{1} y(T), & x^{\prime}(0)=\lambda_{2} y^{\prime}(T), \\
y(0)=\mu_{1} x(T), & y^{\prime}(0)=\mu_{2} x^{\prime}(T),
\end{array}\right.
$$

is equivalent to the system of integral equations

$$
\begin{aligned}
x(t)= & \frac{\mu_{2}}{1-\lambda_{2} \mu_{2}}\left(\frac{\lambda_{1} T\left(\mu_{1} \lambda_{2}+1\right)}{1-\lambda_{1} \mu_{1}}+\lambda_{2} t\right) B_{2}+\frac{\lambda_{2}}{1-\lambda_{2} \mu_{2}}\left(\frac{T\left(\mu_{1}+\mu_{2}\right) \lambda_{1}}{1-\lambda_{1} \mu_{1}}+t\right) A_{2} \\
& +\frac{\lambda_{1}}{1-\lambda_{1} \mu_{1}}\left(A_{1}+\mu_{1} B_{1}\right)+\int_{0}^{t} \frac{(t-s)^{\alpha-1}}{\Gamma(\alpha)} \phi(s) d s
\end{aligned}
$$

and

$$
\begin{aligned}
y(t)= & \frac{\mu_{2}}{1-\lambda_{2} \mu_{2}}\left(\frac{T \mu_{1}\left(\lambda_{1}+\lambda_{2}\right)}{1-\lambda_{1} \mu_{1}}+t\right) B_{2}+\frac{\lambda_{2}}{1-\lambda_{2} \mu_{2}}\left(\frac{T \mu_{1}\left(\lambda_{1} \mu_{2}+1\right)}{1-\lambda_{1} \mu_{1}}+\mu_{2} t\right) A_{2} \\
& +\frac{\mu_{1}}{1-\lambda_{1} \mu_{1}}\left(\lambda_{1} A_{1}+B_{1}\right)+\int_{0}^{t} \frac{(t-s)^{\beta-1}}{\Gamma(\beta)} h(s) d s,
\end{aligned}
$$

where

$$
\begin{array}{ll}
A_{1}=\int_{0}^{T} \frac{(T-s)^{\beta-1}}{\Gamma(\beta)} h(s) d s, & B_{1}=\int_{0}^{T} \frac{(T-s)^{\alpha-1}}{\Gamma(\alpha)} \phi(s) d s, \\
A_{2}=\int_{0}^{T} \frac{(T-s)^{\beta-2}}{\Gamma(\beta-1)} h(s) d s, & B_{2}=\int_{0}^{T} \frac{(T-s)^{\alpha-2}}{\Gamma(\alpha-1)} \phi(s) d s .
\end{array}
$$

Proof We know that the general solution of fractional differential equations in (2.1) can be written as

$$
\begin{aligned}
& x(t)=a_{0}+a_{1} t+\int_{0}^{t} \frac{(t-s)^{\alpha-1}}{\Gamma(\alpha)} \phi(s) d s, \\
& y(t)=b_{0}+b_{1} t+\int_{0}^{t} \frac{(t-s)^{\beta-1}}{\Gamma(\beta)} h(s) d s,
\end{aligned}
$$

where $a_{i}, b_{1}, i=0,1$, are arbitrary real constants.

Using the boundary conditions in (2.4) and (2.5), we have

$$
\begin{aligned}
& x(0)=\lambda_{1} y(T) \quad \Rightarrow \quad a_{0}=\lambda_{1}\left(b_{0}+b_{1} T+A_{1}\right), \\
& y(0)=\mu_{1} x(T) \quad \Rightarrow \quad b_{0}=\mu_{1}\left(a_{0}+a_{1} T+B_{1}\right),
\end{aligned}
$$




$$
\begin{aligned}
& x^{\prime}(0)=\lambda_{2} y^{\prime}(T) \quad \Rightarrow \quad a_{1}=\lambda_{2}\left(b_{1}+A_{2}\right), \\
& y^{\prime}(0)=\mu_{2} x^{\prime}(T) \quad \Rightarrow \quad b_{1}=\mu_{2}\left(a_{1}+B_{2}\right) .
\end{aligned}
$$

From the last two relations we find

$$
a_{1}=\frac{\lambda_{2}}{1-\lambda_{2} \mu_{2}}\left(\mu_{2} B_{2}+A_{2}\right) \quad \text { and } \quad b_{1}=\frac{\mu_{2}}{1-\lambda_{2} \mu_{2}}\left(B_{2}+\lambda_{2} A_{2}\right)
$$

Substituting $a_{1}$ and $b_{1}$ in the first two relations, we find

$$
\begin{aligned}
a_{0} & =\frac{\lambda_{1}}{1-\lambda_{1} \mu_{1}}\left[\mu_{1} T a_{1}+\mu_{1} B_{1}+T b_{1}+A_{1}\right] \\
& =\frac{\lambda_{1}}{1-\lambda_{1} \mu_{1}}\left[\frac{\mu_{1} T \lambda_{2}}{1-\lambda_{2} \mu_{2}}\left(\mu_{2} B_{2}+A_{2}\right)+\mu_{1} B_{1 f}+A_{1}+\frac{T \mu_{2}}{1-\lambda_{2} \mu_{2}}\left(B_{2}+\lambda_{2} A_{2}\right)\right] \\
& =\frac{\lambda_{1}}{1-\lambda_{1} \mu_{1}}\left[\frac{T \mu_{2}\left(\mu_{1} \lambda_{2}+1\right)}{1-\lambda_{2} \mu_{2}} B_{2}+\frac{T \lambda_{2}\left(\mu_{1}+\mu_{2}\right)}{1-\lambda_{2} \mu_{2}} A_{2}+A_{1}+\mu_{1} B_{1}\right],
\end{aligned}
$$

and

$$
\begin{aligned}
b_{0}= & \mu_{1} a_{0}+\mu_{1} a_{1} T+\mu_{1} B_{1} \\
= & \frac{\lambda_{1} \mu_{1}}{1-\lambda_{1} \mu_{1}}\left[\frac{T \mu_{2}\left(\mu_{1} \lambda_{2}+1\right)}{1-\lambda_{2} \mu_{2}} B_{2}+\frac{T \lambda_{2}\left(\mu_{1}+\mu_{2}\right)}{1-\lambda_{2} \mu_{2}} A_{2}+A_{1}+\mu_{1} B_{1}\right] \\
& +\frac{\mu_{1} T \lambda_{2}}{1-\lambda_{2} \mu_{2}}\left[\mu_{2} B_{2}+A_{2}\right]+\mu_{1} B_{1} \\
= & \frac{T \mu_{1} \mu_{2}}{1-\lambda_{2} \mu_{2}}\left(\frac{\lambda_{1}+\lambda_{2}}{1-\lambda_{1} \mu_{1}}\right) B_{2}+\frac{T \mu_{1} \lambda_{2}}{1-\lambda_{2} \mu_{2}}\left(\frac{\lambda_{1} \mu_{2}+1}{1-\lambda_{1} \mu_{1}}\right) A_{2} \\
& +\frac{\lambda_{1} \mu_{1}}{1-\lambda_{1} \mu_{1}} A_{1}+\frac{\mu_{1}}{1-\lambda_{1} \mu_{1}} B_{1} .
\end{aligned}
$$

Inserting the values of $a_{i}, b_{i}, i=0,1$, in (2.4) and (2.5), we get solutions (2.2) and (2.3). The converse follows by direct computation. This completes the proof.

\section{Main results}

Let us introduce the space $X=\{u(t) \mid u(t) \in C([0, T], \mathbb{R})\}$ endowed with the norm $\|u\|=$ $\sup \{|u(t)|, t \in[0, T]\}$. Obviously, $(X,\|\cdot\|)$ is a Banach space. Then the product space $(X \times$ $X,\|(u, v)\|)$ is also a Banach space equipped with the norm $\|(u, v)\|=\|u\|+\|v\|$.

In view of Lemma 2.5, we define the operator $T: X \times X \rightarrow X \times X$ by

$$
T(u, v)(t)=\left(\begin{array}{l}
T_{1}(u, v)(t) \\
T_{2}(u, v)(t)
\end{array}\right),
$$

where

$$
\begin{aligned}
& T_{1}(u, v)(t) \\
& =\frac{\mu_{2}}{1-\lambda_{2} \mu_{2}}\left(\frac{\lambda_{1} T\left(\mu_{1} \lambda_{2}+1\right)}{1-\lambda_{1} \mu_{1}}+\lambda_{2} t\right) B_{2 f}+\frac{\lambda_{2}}{1-\lambda_{2} \mu_{2}}\left(\frac{T\left(\mu_{1}+\mu_{2}\right) \lambda_{1}}{1-\lambda_{1} \mu_{1}}+t\right) A_{2 g} \\
& \quad+\frac{\lambda_{1}}{1-\lambda_{1} \mu_{1}}\left(A_{1 g}+\mu_{1} B_{1 f}\right)+\int_{0}^{t} \frac{(t-s)^{\alpha-1}}{\Gamma(\alpha)} f(s, x(s), y(s)) d s,
\end{aligned}
$$


and

$$
\begin{aligned}
T_{2}(u, v)(t) & \\
= & \frac{\mu_{2}}{1-\lambda_{2} \mu_{2}}\left(\frac{T \mu_{1}\left(\lambda_{1}+\lambda_{2}\right)}{1-\lambda_{1} \mu_{1}}+t\right) B_{2 f}+\frac{\lambda_{2}}{1-\lambda_{2} \mu_{2}}\left(\frac{T \mu_{1}\left(\lambda_{1} \mu_{2}+1\right)}{1-\lambda_{1} \mu_{1}}+\mu_{2} t\right) A_{2 g} \\
& +\frac{\mu_{1}}{1-\lambda_{1} \mu_{1}}\left(\lambda_{1} A_{1 g}+B_{1 f}\right)+\int_{0}^{t} \frac{(t-s)^{\beta-1}}{\Gamma(\beta)} g(s, x(s), y(s)) d s, \\
A_{1 g}= & \int_{0}^{T} \frac{(T-s)^{\beta-1}}{\Gamma(\beta)} g(s, x(s), y(s)) d s, \quad B_{1 f}=\int_{0}^{T} \frac{(T-s)^{\alpha-1}}{\Gamma(\alpha)} f(s, x(s), y(s)) d s, \\
A_{2 g}= & \int_{0}^{T} \frac{(T-s)^{\beta-2}}{\Gamma(\beta-1)} g(s, x(s), y(s)) d s, \quad B_{2 f}=\int_{0}^{T} \frac{(T-s)^{\alpha-2}}{\Gamma(\alpha-1)} f(s, x(s), y(s)) d s .
\end{aligned}
$$

For convenience, we put

$$
\begin{aligned}
M_{1}= & \frac{\left|\mu_{2}\right|}{\left|1-\lambda_{2} \mu_{2}\right|}\left(\frac{\left|\lambda_{1}\right|\left(\left|\mu_{1}\right|\left|\lambda_{2}\right|+1\right)}{\left|1-\lambda_{1} \mu_{1}\right|}+\left|\lambda_{2}\right|\right) \frac{T^{\alpha}}{\Gamma(\alpha)} \\
& +\left(\frac{\left|\lambda_{1}\right|\left|\mu_{1}\right|}{\left|1-\lambda_{1} \mu_{1}\right|}+1\right) \frac{T^{\alpha}}{\Gamma(\alpha+1)}, \\
M_{2}= & \frac{\left|\lambda_{2}\right|}{\left|1-\lambda_{2} \mu_{2}\right|}\left(\frac{\left(\left|\mu_{1}\right|+\left|\mu_{2}\right|\right)\left|\lambda_{1}\right|}{\left|1-\lambda_{1} \mu_{1}\right|}+1\right) \frac{T^{\beta}}{\Gamma(\beta)}+\frac{\left|\lambda_{1}\right|}{\left|1-\lambda_{1} \mu_{1}\right|} \frac{T^{\beta}}{\Gamma(\beta+1)}, \\
M_{3}= & \frac{\left|\mu_{2}\right|}{\left|1-\lambda_{2} \mu_{2}\right|}\left(\frac{\left|\mu_{1}\right|\left(\left|\lambda_{1}\right|+\left|\lambda_{2}\right|\right)}{\left|1-\lambda_{1} \mu_{1}\right|}+1\right) \frac{T^{\alpha}}{\Gamma(\alpha)}+\frac{\left|\mu_{1}\right|}{\left|1-\lambda_{1} \mu_{1}\right|} \frac{T^{\alpha}}{\Gamma(\alpha+1)}, \\
M_{4}= & \frac{\left|\lambda_{2}\right|}{\left|1-\lambda_{2} \mu_{2}\right|}\left(\frac{\left|\mu_{1}\right|\left(\left|\lambda_{1}\right|\left|\mu_{2}\right|+1\right)}{\left|1-\lambda_{1} \mu_{1}\right|}+\left|\mu_{2}\right|\right) \frac{T^{\beta}}{\Gamma(\beta)} \\
& +\left(\frac{\left|\lambda_{1}\right|\left|\mu_{1}\right|}{\left|1-\lambda_{1} \mu_{1}\right|}+1\right) \frac{T^{\beta}}{\Gamma(\beta+1)} .
\end{aligned}
$$

In the first result, we prove the existence and uniqueness of solutions of boundary value problem (1.1)-(1.2) via Banach's contraction principle.

Theorem 3.1 Assume that:

$\left(\mathrm{H}_{1}\right) f, g:[0, T] \times \mathbb{R} \times \mathbb{R} \rightarrow \mathbb{R}$ are continuous functions and there exist positive constants $\ell_{1}$ and $\ell_{2}$ such that for all $t \in[0, T]$ and $x_{i}, y_{i} \in \mathbb{R}, i=1,2$, we have

$$
\begin{aligned}
& \left|f\left(t, x_{1}, x_{2}\right)-f\left(t, y_{1}, y_{2}\right)\right| \leq \ell_{1}\left(\left|x_{1}-y_{1}\right|+\left|x_{2}-y_{2}\right|\right), \\
& \left|g\left(t, x_{1}, x_{2}\right)-g\left(t, y_{1}, y_{2}\right)\right| \leq \ell_{2}\left(\left|x_{1}-y_{1}\right|+\left|x_{2}-y_{2}\right|\right) .
\end{aligned}
$$

If

$$
\left(M_{1}+M_{3}\right) \ell_{1}+\left(M_{2}+M_{4}\right) \ell_{2}<1
$$

where $M_{i}, i=1,2,3,4$, are given by (3.1)-(3.4), then system (1.1)-(1.2) has a unique solution. 
Proof Define $\sup _{t \in[0, T]} f(t, 0,0)=N_{1}<\infty$ and $\sup _{t \in[0, T]} g(t, 0,0)=N_{2}<\infty$ and $r>0$ such that

$$
r>\frac{\left(M_{1}+M_{3}\right) N_{1}+\left(M_{2}+M_{4}\right) N_{2}}{1-\left(M_{1}+M_{3}\right) \ell_{1}-\left(M_{2}+M_{4}\right) \ell_{2}} .
$$

We show that $T B_{r} \subset B_{r}$, where $B_{r}=\{(u, v) \in X \times X:\|(u, v)\| \leq r\}$.

By assumption $\left(\mathrm{H}_{1}\right)$, for $(u, v) \in B_{r}, t \in[0, T]$, we have

$$
\begin{aligned}
|f(t, u(t), v(t))| & \leq|f(t, u(t), v(t))-f(t, 0,0)|+|f(t, 0,0)| \\
& \leq \ell_{1}(|u(t)|+|v(t)|)+N_{1} \\
& \leq \ell_{1}(\|u\|+\|v\|)+N_{1} \leq \ell_{1} r+N_{1}
\end{aligned}
$$

and

$$
|g(t, u(t), v(t))| \leq \ell_{2}(\|u\|+\|v\|)+N_{2} \leq \ell_{2} r+N_{2}
$$

which lead to

$$
\begin{aligned}
\left|T_{1}(u, v)(t)\right| \leq & \frac{\left|\mu_{2}\right|}{\left|1-\lambda_{2} \mu_{2}\right|}\left(\frac{\left|\lambda_{1}\right|\left(\left|\mu_{1}\right|\left|\lambda_{2}\right|+1\right)}{\left|1-\lambda_{1} \mu_{1}\right|}+\left|\lambda_{2}\right|\right) \frac{T^{\alpha}}{\Gamma(\alpha)}\left(\ell_{1} r+N_{1}\right) \\
& +\frac{\left|\lambda_{2}\right|}{\left|1-\lambda_{2} \mu_{2}\right|}\left(\frac{\left(\left|\mu_{1}\right|+\left|\mu_{2}\right|\right)\left|\lambda_{1}\right|}{\left|1-\lambda_{1} \mu_{1}\right|}+1\right) \frac{T^{\beta}}{\Gamma(\beta)}\left(\ell_{2} r+N_{2}\right) \\
& +\frac{\left|\lambda_{1}\right|}{\left|1-\lambda_{1} \mu_{1}\right|}\left(\frac{T^{\beta}}{\Gamma(\beta+1)}\left(\ell_{2} r+N_{2}\right)+\left|\mu_{1}\right| \frac{T^{\alpha}}{\Gamma(\alpha+1)}\left(\ell_{1} r+N_{1}\right)\right) \\
& +\frac{T^{\alpha}}{\Gamma(\alpha+1)}\left(\ell_{1} r+N_{1}\right) \\
= & \left(M_{1} \ell_{1}+M_{2} \ell_{2}\right) r+M_{1} N_{1}+M_{2} N_{2} .
\end{aligned}
$$

Hence

$$
\left\|T_{1}(u, v)\right\| \leq\left(M_{1} \ell_{1}+M_{2} \ell_{2}\right) r+M_{1} N_{1}+M_{2} N_{2}
$$

In the same way, we can obtain that

$$
\left\|T_{2}(u, v)\right\| \leq\left(M_{3} \ell_{1}+M_{4} \ell_{2}\right) r+M_{3} N_{1}+M_{4} N_{2} .
$$

Consequently,

$$
\|T(u, v)\| \leq\left[\left(M_{1}+M_{3}\right) \ell_{1}+\left(M_{2}+M_{4}\right) \ell_{2}\right] r+\left(M_{1}+M_{3}\right) N_{1}+\left(M_{2}+M_{4}\right) N_{2} \leq r .
$$

Now, for $\left(u_{2}, v_{2}\right),\left(u_{1}, v_{1}\right) \in X \times X$ and for any $t \in[0, T]$, we get

$$
\begin{aligned}
& \left|T_{1}\left(u_{2}, v_{2}\right)(t)-T_{1}\left(u_{1}, v_{1}\right)(t)\right| \\
& \quad \leq \frac{\left|\mu_{2}\right|}{\left|1-\lambda_{2} \mu_{2}\right|}\left(\frac{\left|\lambda_{1}\right|\left(\left|\mu_{1}\right|\left|\lambda_{2}\right|+1\right)}{\left|1-\lambda_{1} \mu_{1}\right|}+\left|\lambda_{2}\right|\right) \frac{T^{\alpha}}{\Gamma(\alpha)} \ell_{1}\left(\left\|u_{2}-u_{1}\right\|+\left\|v_{2}-v_{1}\right\|\right)
\end{aligned}
$$




$$
\begin{aligned}
& +\frac{\left|\lambda_{2}\right|}{\left|1-\lambda_{2} \mu_{2}\right|}\left(\frac{\left(\left|\mu_{1}\right|+\left|\mu_{2}\right|\right)\left|\lambda_{1}\right|}{\left|1-\lambda_{1} \mu_{1}\right|}+1\right) \frac{T^{\beta}}{\Gamma(\beta)} \ell_{2}\left(\left\|u_{2}-u_{1}\right\|+\left\|v_{2}-v_{1}\right\|\right) \\
& +\frac{\left|\lambda_{1}\right|}{\left|1-\lambda_{1} \mu_{1}\right|} \frac{T^{\beta}}{\Gamma(\beta+1)} \ell_{2}\left(\left\|u_{2}-u_{1}\right\|+\left\|v_{2}-v_{1}\right\|\right) \\
& +\frac{\left|\lambda_{1}\right|\left|\mu_{1}\right|}{\left|1-\lambda_{1} \mu_{1}\right|} \frac{T^{\alpha}}{\Gamma(\alpha+1)} \ell_{1}\left(\left\|u_{2}-u_{1}\right\|+\left\|v_{2}-v_{1}\right\|\right) \\
& +\frac{T^{\alpha}}{\Gamma(\alpha+1)} \ell_{1}\left(\left\|u_{2}-u_{1}\right\|+\left\|v_{2}-v_{1}\right\|\right) \\
& =\left(M_{1} \ell_{1}+M_{2} \ell_{2}\right)\left(\left\|u_{2}-u_{1}\right\|+\left\|v_{2}-v_{1}\right\|\right),
\end{aligned}
$$

and consequently we obtain

$$
\left\|T_{1}\left(u_{2}, v_{2}\right)-T_{1}\left(u_{1}, v_{1}\right)\right\| \leq\left(M_{1} \ell_{1}+M_{2} \ell_{2}\right)\left(\left\|u_{2}-u_{1}\right\|+\left\|v_{2}-v_{1}\right\|\right) .
$$

Similarly,

$$
\left\|T_{2}\left(u_{2}, v_{2}\right)(t)-T_{2}\left(u_{1}, v_{1}\right)\right\| \leq\left(M_{3} \ell_{1}+M_{4} \ell_{2}\right)\left(\left\|u_{2}-u_{1}\right\|+\left\|v_{2}-v_{1}\right\|\right)
$$

It follows from (3.5) and (3.6) that

$$
\left\|T\left(u_{2}, v_{2}\right)-T\left(u_{1}, v_{1}\right)\right\| \leq\left[\left(M_{1}+M_{3}\right) \ell_{1}+\left(M_{2}+M_{4}\right) \ell_{2}\right]\left(\left\|u_{2}-u_{1}\right\|+\left\|v_{2}-v_{1}\right\|\right) .
$$

Since $\left(M_{1}+M_{3}\right) \ell_{1}+\left(M_{2}+M_{4}\right) \ell_{2}<1$, therefore, $T$ is a contraction operator. So, by Banach's fixed point theorem, the operator $T$ has a unique fixed point, which is the unique solution of problem (1.1)-(1.2). This completes the proof.

The second result is based on the Leray-Schauder alternative.

Lemma 3.2 (Leray-Schauder alternative [28], p.4) Let $F: E \rightarrow$ E be a completely continuous operator (i.e., a map restricted to any bounded set in $E$ is compact). Let

$$
\mathcal{E}(F)=\{x \in E: x=\lambda F(x) \text { for some } 0<\lambda<1\} .
$$

Then either the set $\mathcal{E}(F)$ is unbounded or $F$ has at least one fixed point.

Theorem 3.3 Assume that:

$\left(\mathrm{H}_{3}\right) f, g:[0, T] \times \mathbb{R} \times \mathbb{R} \rightarrow \mathbb{R}$ are continuous functions and there exist real constants $k_{i}, \gamma_{i} \geq$ $0(i=0,1,2)$ and $k_{0}>0, \gamma_{0}>0$ such that $\forall x_{i} \in \mathbb{R}(i=1,2)$, we have

$$
\begin{aligned}
& \left|f\left(t, x_{1}, x_{2}\right)\right| \leq k_{0}+k_{1}\left|x_{1}\right|+k_{2}\left|x_{2}\right|, \\
& \left|g\left(t, x_{1}, x_{2}\right)\right| \leq \gamma_{0}+\gamma_{1}\left|x_{1}\right|+\gamma_{2}\left|x_{2}\right| .
\end{aligned}
$$

If

$$
\left(M_{1}+M_{3}\right) k_{1}+\left(M_{2}+M_{4}\right) \gamma_{1}<1 \text { and }\left(M_{1}+M_{3}\right) k_{2}+\left(M_{2}+M_{4}\right) \gamma_{2}<1 \text {, }
$$


where $M_{i}, i=1,2,3,4$, are given by (3.1)-(3.4), then system (1.1)-(1.2) has at least one solution.

Proof First we show that the operator $T: X \times X \rightarrow X \times X$ is completely continuous. By the continuity of functions $f$ and $g$, the operator $T$ is continuous.

Let $\Omega \subset X \times X$ be bounded. Then there exist positive constants $L_{1}$ and $L_{2}$ such that

$$
|f(t, u(t), v(t))| \leq L_{1}, \quad|h(t, u(t), v(t))| \leq L_{2}, \quad \forall(u, v) \in \Omega .
$$

Then, for any $(u, v) \in \Omega$, we have

$$
\begin{aligned}
\left|T_{1}(u, v)(t)\right| \leq & \frac{\left|\mu_{2}\right|}{\left|1-\lambda_{2} \mu_{2}\right|}\left(\frac{\left|\lambda_{1}\right|\left(\left|\mu_{1}\right|\left|\lambda_{2}\right|+1\right)}{\left|1-\lambda_{1} \mu_{1}\right|}+\left|\lambda_{2}\right|\right) \frac{T^{\alpha}}{\Gamma(\alpha)} L_{1} \\
& +\frac{\left|\lambda_{2}\right|}{\left|1-\lambda_{2} \mu_{2}\right|}\left(\frac{\left(\left|\mu_{1}\right|+\left|\mu_{2}\right|\right)\left|\lambda_{1}\right|}{\left|1-\lambda_{1} \mu_{1}\right|}+1\right) \frac{T^{\beta}}{\Gamma(\beta)} L_{2} \\
& +\frac{\left|\lambda_{1}\right|}{\left|1-\lambda_{1} \mu_{1}\right|}\left(\frac{T^{\beta}}{\Gamma(\beta+1)} L_{2}+\left|\mu_{1}\right| \frac{T^{\alpha}}{\Gamma(\alpha+1)} L_{1}\right)+\frac{T^{\alpha}}{\Gamma(\alpha+1)} L_{1} \\
= & M_{1} L_{1}+M_{2} L_{2},
\end{aligned}
$$

which implies that

$$
\left\|T_{1}(u, v)\right\| \leq M_{1} L_{1}+M_{2} L_{2}
$$

Similarly, we get

$$
\left\|T_{2}(u, v)\right\| \leq M_{3} L_{1}+M_{4} L_{2}
$$

Thus, it follows from the above inequalities that the operator $T$ is uniformly bounded, since $\|T(u, v)\| \leq\left(M_{1}+M_{3}\right) L_{1}+\left(M_{2}+M_{4}\right) L_{2}$.

Next, we show that $T$ is equicontinuous. Let $t_{1}, t_{2} \in[0, T]$ with $t_{1}<t_{2}$. Then we have

$$
\begin{aligned}
\mid & T_{1}\left(u\left(t_{2}\right), v\left(t_{2}\right)\right)-T_{1}\left(u\left(t_{1}\right), v\left(t_{1}\right)\right) \mid \\
\leq & \frac{L_{1}\left|\lambda_{2}\right|\left|\mu_{2}\right|}{\left|1-\lambda_{2} \mu_{2}\right|}\left(t_{2}-t_{1}\right)+\frac{L_{2}\left|\lambda_{2}\right|}{\left|1-\lambda_{2} \mu_{2}\right|}\left(t_{2}-t_{1}\right) \\
& +L_{1}\left|\frac{1}{\Gamma(\alpha)} \int_{0}^{t_{2}}\left(t_{2}-s\right)^{\alpha-1} d s-\frac{1}{\Gamma(\alpha)} \int_{0}^{t_{1}}\left(t_{1}-s\right)^{\alpha-1} d s\right| \\
\leq & \frac{L_{1}\left|\lambda_{2}\right|\left|\mu_{2}\right|}{\left|1-\lambda_{2} \mu_{2}\right|}\left(t_{2}-t_{1}\right)+\frac{L_{2}\left|\lambda_{2}\right|}{\left|1-\lambda_{2} \mu_{2}\right|}\left(t_{2}-t_{1}\right) \\
& +L_{1}\left\{\frac{1}{\Gamma(\alpha)} \int_{0}^{t_{1}}\left[\left(t_{2}-s\right)^{\alpha-1}-\left(t_{1}-s\right)^{\alpha-1}\right] d s+\frac{1}{\Gamma(q)} \int_{t_{1}}^{t_{2}}\left(t_{2}-s\right)^{q-1} d s\right\} \\
\leq & \frac{L_{1}\left|\lambda_{2}\right|\left|\mu_{2}\right|}{\left|1-\lambda_{2} \mu_{2}\right|}\left(t_{2}-t_{1}\right)+\frac{L_{2}\left|\lambda_{2}\right|}{\left|1-\lambda_{2} \mu_{2}\right|}\left(t_{2}-t_{1}\right) \\
& +\frac{L_{1}}{\Gamma(\alpha+1)}\left[2\left(t_{2}-t_{1}\right)^{\alpha}+\left|t_{2}^{\alpha}-t_{1}^{\alpha}\right|\right] .
\end{aligned}
$$


Analogously, we can obtain

$$
\begin{aligned}
& \left|T_{2}\left(u\left(t_{2}\right), v\left(t_{2}\right)\right)-T_{2}\left(u\left(t_{1}\right), v\left(t_{1}\right)\right)\right| \\
& \quad \leq \frac{L_{1}\left|\mu_{2}\right|}{\left|1-\lambda_{2} \mu_{2}\right|}\left(t_{2}-t_{1}\right)+\frac{L_{2}\left|\lambda_{2}\right|\left|\mu_{2}\right|}{\left|1-\lambda_{2} \mu_{2}\right|}\left(t_{2}-t_{1}\right)+\frac{L_{2}}{\Gamma(\beta+1)}\left[2\left(t_{2}-t_{1}\right)^{\beta}+\left|t_{2}^{\beta}-t_{1}^{\beta}\right|\right] .
\end{aligned}
$$

Therefore, the operator $T(u, v)$ is equicontinuous, and thus the operator $T(u, v)$ is completely continuous.

Finally, it will be verified that the set $\mathcal{E}=\{(u, v) \in X \times X \mid(u, v)=\lambda T(u, v), 0 \leq \lambda \leq 1\}$ is bounded. Let $(u, v) \in \mathcal{E}$, with $(u, v)=\lambda T(u, v)$. For any $t \in[0, T]$, we have

$$
u(t)=\lambda T_{1}(u, v)(t), \quad v(t)=\lambda T_{2}(u, v)(t)
$$

Then

$$
\begin{aligned}
|u(t)| & \leq M_{1}\left(k_{0}+k_{1}|u|+k_{2}|v|\right)+M_{2}\left(\gamma_{0}+\gamma_{1}|u|+\gamma_{2}|v|\right) \\
& =M_{1} k_{0}+M_{2} \gamma_{0}+\left(M_{1} k_{1}+M_{2} \gamma_{1}\right)|u|+\left(M_{1} k_{2}+M_{2} \gamma_{2}\right)|v|
\end{aligned}
$$

and

$$
\begin{aligned}
|v(t)| & \leq M_{3}\left(k_{0}+k_{1}|u|+k_{2}|v|\right)+M_{4}\left(\gamma_{0}+\gamma_{1}|u|+\gamma_{2}|v|\right) \\
& =M_{3} k_{0}+M_{4} \gamma_{0}+\left(M_{3} k_{1}+M_{4} \gamma_{1}\right)|u|+\left(M_{3} k_{2}+M_{4} \gamma_{2}\right)|v| .
\end{aligned}
$$

Hence we have

$$
\|u\| \leq M_{1} k_{0}+M_{2} \gamma_{0}+\left(M_{1} k_{1}+M_{2} \gamma_{1}\right)\|u\|+\left(M_{1} k_{2}+M_{2} \gamma_{2}\right)\|v\|
$$

and

$$
\|v\| \leq M_{3} k_{0}+M_{4} \gamma_{0}+\left(M_{3} k_{1}+M_{4} \gamma_{1}\right)\|u\|+\left(M_{3} k_{2}+M_{4} \gamma_{2}\right)\|v\|,
$$

which imply that

$$
\begin{aligned}
\|u\|+\|v\| \leq & \left(M_{1}+M_{3}\right) k_{0}+\left(M_{2}+M_{4}\right) \gamma_{0}+\left[\left(M_{1}+M_{3}\right) k_{1}+\left(M_{2}+M_{4}\right) \gamma_{1}\right]\|u\| \\
& +\left[\left(M_{1}+M_{3}\right) k_{2}+\left(M_{2}+M_{4}\right) \gamma_{2}\right]\|v\| .
\end{aligned}
$$

Consequently,

$$
\|(u, v)\| \leq \frac{\left(M_{1}+M_{3}\right) k_{0}+\left(M_{2}+M_{4}\right) \gamma_{0}}{M_{0}},
$$

where $M_{0}=\min \left\{1-\left[\left(M_{1}+M_{3}\right) k_{1}+\left(M_{2}+M_{4}\right) \gamma_{1}\right], 1-\left[\left(M_{1}+M_{3}\right) k_{2}+\left(M_{2}+M_{4}\right) \gamma_{2}\right]\right\}$, which proves that $\mathcal{E}$ is bounded. Thus, by Lemma 3.2, the operator $T$ has at least one fixed point. Hence boundary value problem (1.1)-(1.2) has at least one solution. The proof is complete. 
Example 3.4 Consider the following system of fractional boundary value problem:

$$
\left\{\begin{array}{l}
{ }^{\mathrm{c}} D^{3 / 2} x(t)=\frac{1}{4(t+2)^{2}} \frac{|x(t)|}{1+|x(t)|}+1+\frac{1}{32} \sin ^{2} y(t)+\frac{1}{\sqrt{t^{2}+1}}, \quad t \in[0,1] \\
{ }^{\mathrm{c}} D^{3 / 2} y(t)=\frac{1}{32 \pi} \sin (2 \pi x(t))+\frac{|y(t)|}{16(1+\mid y(t))}+\frac{1}{2}, \quad t \in[0,1], \\
x(0)=\frac{1}{2} y(1), \quad x^{\prime}(0)=\frac{1}{3} y^{\prime}(1), \\
y(0)=\frac{4}{5} x(1), \quad y^{\prime}(0)=\frac{3}{4} x^{\prime}(1) .
\end{array}\right.
$$

Here $\alpha=\beta=3 / 2, \lambda_{1}=1 / 2, \lambda_{2}=1 / 3, \mu_{1}=4 / 5, \mu_{2}=3 / 4, f(t, u, v)=\frac{1}{4(t+2)^{2}} \frac{|u|}{1+|u|}+1+\frac{1}{32} \sin ^{2} v$, and $h(t, u, v)=\frac{1}{32 \pi} \sin (2 \pi u)+\frac{|v|}{16(1+|v|)}+\frac{1}{2}$. With the given data, we find that $M_{1} \approx 2.8216701$, $M_{2} \approx 1.4892147, M_{3} \approx 3.3860044, M_{4} \approx 2.5499538$. Note that $\left|f\left(t, u_{1}, u_{2}\right)-f\left(t, v_{1}, v_{2}\right)\right| \leq$ $\frac{1}{16}\left|u_{1}-u_{2}\right|+\frac{1}{16}\left|v_{1}-v_{2}\right|,\left|g\left(t, u_{1}, u_{2}\right)-g\left(t, v_{1}, v_{2}\right)\right| \leq \frac{1}{16}\left|u_{1}-u_{2}\right|+\frac{1}{16}\left|v_{1}-v_{2}\right|$, and $\left(M_{1}+M_{3}\right) \ell_{1}+$ $\left(M_{2}+M_{4}\right) \ell_{2} \approx 0.6404276<1$. Thus all the conditions of Theorem 3.1 are satisfied, and consequently, its conclusion applies to problem (3.7).

\section{Conclusions}

In this work, we have established the existence and uniqueness results for a nonlinear coupled system of Caputo type fractional differential equations supplemented with nonseparated coupled boundary conditions. Our results are not only new in the given setting but also lead to some new interesting situations for specific values of the parameters involved in the problem. For instance, if we choose $\lambda_{1}=1=\lambda_{2}$ and $\mu_{1}=-1=\mu_{2}$ or vice versa, our results correspond to a boundary value problem of nonlinear coupled fractional differential equations subject to a combination of coupled periodic and anti-periodic boundary conditions of the form: $x(0)=y(T), x^{\prime}(0)=y^{\prime}(T), y(0)=-x(T)$, $y^{\prime}(0)=-x^{\prime}(T)$ or $x(0)=-y(T), x^{\prime}(0)=-y^{\prime}(T), y(0)=x(T), y^{\prime}(0)=x^{\prime}(T)$. Further, by tak ing $\lambda_{1}=1=-\lambda_{2}$ and $-\mu_{1}=1=\mu_{2}$, we obtain the results for the coupled system with the boundary conditions: $x(0)=y(T), x^{\prime}(0)=-y^{\prime}(T), y(0)=-x(T), y^{\prime}(0)=x^{\prime}(T)$, while the results for the boundary conditions $x(0)=-y(T), x^{\prime}(0)=y^{\prime}(T), y(0)=x(T), y^{\prime}(0)=-x^{\prime}(T)$ can be obtained by letting $-\lambda_{1}=1=\lambda_{2}$ and $\mu_{1}=1=-\mu_{2}$. In the case of $x(0)=0, y(0)=0$ $\left(\lambda_{1}=0=\mu_{1}\right), x^{\prime}(0)=\lambda_{2} y^{\prime}(T), y^{\prime}(0)=\mu_{2} x^{\prime}(T)$, our results correspond to a problem with coupled flux type conditions. Thus, the work established in this article is of quite a general nature and covers a variety of special cases associated with particular values of the parameters involved in the problem.

\section{Acknowledgements}

This project was funded by the Deanship of Scientific Research (DSR) at King Abdulaziz University, Jeddah, under grant No. (14-130-36-RG). The authors, therefore, acknowledge with thanks DSR for technical and financial support. We also thank the reviewers for their useful comments that led to the improvement of the original manuscript.

\section{Funding}

This research was funded by the Deanship of Scientific Research (DSR) at King Abdulaziz University, Jeddah, under grant No. (14-130-36-RG).

Ethics approval and consent to participate

The research has been carried out within an appropriate ethical framework and each of the authors, HHA, SKN, RPA, BA and $A A$, participated in this research work.

\section{Competing interests}

The authors declare that they have no competing interests. 
Authors' contributions

Each of the authors, HHA, SKN, RPA, BA and AA, contributed to each part of this work equally. All authors read and approved the final manuscript.

\section{Author details}

${ }^{1}$ Nonlinear Analysis and Applied Mathematics (NAAM) - Research Group, Department of Mathematics, Faculty of Science, King Abdulaziz University, P.O. Box 80203, Jeddah, 21589, Saudi Arabia. ${ }^{2}$ Department of Mathematics, University of loannina, loannina, 451 10, Greece. ${ }^{3}$ Department of Mathematics, Texas A\&M University, Kingsville, TX 78363-8202, USA

\section{Publisher's Note}

Springer Nature remains neutral with regard to jurisdictional claims in published maps and institutional affiliations.

Received: 4 January 2017 Accepted: 1 May 2017 Published online: 12 May 2017

\section{References}

1. Podlubny, I: Fractional Differential Equations. Academic Press, San Diego (1999)

2. Kilbas, AA, Srivastava, HM, Trujillo, JJ: Theory and Applications of Fractional Differential Equations. North-Holland Mathematics Studies, vol. 204. Elsevier, Amsterdam (2006)

3. Miller, KS, Ross, B: An Introduction to the Fractional Calculus and Fractional Differential Equations. Wiley, New York (1993)

4. Sabatier, J, Agrawal, OP, Machado, JAT (eds.): Advances in Fractional Calculus: Theoretical Developments and Applications in Physics and Engineering. Springer, Dordrecht (2007)

5. Liang, S, Zhang, J: Existence of multiple positive solutions for $m$-point fractional boundary value problems on an infinite interval. Math. Comput. Model. 54, 1334-1346 (2011)

6. Goodrich, C: Existence and uniqueness of solutions to a fractional difference equation with nonlocal conditions. Comput. Math. Appl. 61, 191-202 (2011)

7. Wang, JR, Zhou, Y, Feckan, M: On recent developments in the theory of boundary value problems for impulsive fractional differential equations. Comput. Math. Appl. 64, 3008-3020 (2012)

8. Ahmad, B: On nonlocal boundary value problems for nonlinear integro-differential equations of arbitrary fractional order. Results Math. 63, 183-194 (2013)

9. Zhai, C, Xu, L: Properties of positive solutions to a class of four-point boundary value problem of Caputo fractional differential equations with a parameter. Commun. Nonlinear Sci. Numer. Simul. 19, 2820-2827 (2014)

10. Henderson, J, Kosmatov, N: Eigenvalue comparison for fractional boundary value problems with the Caputo derivative. Fract. Calc. Appl. Anal. 17, 872-880 (2014)

11. Ding, Y, Wei, Z, Xu, J, O'Regan, D: Extremal solutions for nonlinear fractional boundary value problems with p-Laplacian. J. Comput. Appl. Math. 288, 151-158 (2015)

12. Wang, H: Existence of solutions for fractional anti-periodic BVP. Results Math. 68, 227-245 (2015)

13. Ahmad, B, Ntouyas, SK: Some fractional-order one-dimensional semi-linear problems under nonlocal integral boundary conditions. Rev. R. Acad. Cienc. Exactas Fís. Nat., Ser. A Mat. 110, 159-172 (2016)

14. Sokolov, IM, Klafter, J, Blumen, A: Fractional kinetics. Phys. Today 55, 48-54 (2002)

15. Petras, I, Magin, RL: Simulation of drug uptake in a two compartmental fractional model for a biological system. Commun. Nonlinear Sci. Numer. Simul. 16, 4588-4595 (2011)

16. Ding, Y, Wang, Z, Ye, H: Optimal control of a fractional-order HIV-immune system with memory. IEEE Trans. Control Syst. Technol. 20, 763-769 (2012)

17. Carvalho, A, Pinto, CMA: A delay fractional order model for the co-infection of malaria and HIV/AIDS. Int. J. Dyn. Control (2016). doi:10.1007/s40435-016-0224-3

18. Javidi, M, Ahmad, B: Dynamic analysis of time fractional order phytoplankton-toxic phytoplankton-zooplankton system. Ecol. Model. 318, 8-18 (2015)

19. Ge, ZM, Ou, CY: Chaos synchronization of fractional order modified Duffing systems with parameters excited by a chaotic signal. Chaos Solitons Fractals 35, 705-717 (2008)

20. Faieghi, M, Kuntanapreeda, S, Delavari, H, Baleanu, D: LMI-based stabilization of a class of fractional-order chaotic systems. Nonlinear Dyn. 72, 301-309 (2013)

21. Zhang, F, Chen, G, Li, C, Kurths, J: Chaos synchronization in fractional differential systems. Philos. Trans. R. Soc. Lond. A $371,20120155(2013)$

22. Senol, B, Yeroglu, C: Frequency boundary of fractional order systems with nonlinear uncertainties. J. Franklin Inst. 350 1908-1925 (2013)

23. Henderson, J, Luca, R: Nonexistence of positive solutions for a system of coupled fractional boundary value problems. Bound. Value Probl. 2015, 138 (2015)

24. Ahmad, B, Ntouyas, SK: Existence results for a coupled system of Caputo type sequential fractional differential equations with nonlocal integral boundary conditions. Appl. Math. Comput. 266, 615-622 (2015)

25. Wang, JR, Zhang, Y: Analysis of fractional order differential coupled systems. Math. Methods Appl. Sci. 38, 3322-3338 (2015)

26. Tariboon, J, Ntouyas, SK, Sudsutad, W: Coupled systems of Riemann-Liouville fractional differential equations with Hadamard fractional integral boundary conditions. J. Nonlinear Sci. Appl. 9, 295-308 (2016)

27. Ahmad, B, Ntouyas, SK, Alsaedi, A: On a coupled system of fractional differential equations with coupled nonlocal and integral boundary conditions. Chaos Solitons Fractals 83, 234-241 (2016)

28. Granas, A, Dugundji, J: Fixed Point Theory. Springer, New York (2005) 